\title{
The red and blue galaxy populations in the GOODS field: evidence for an excess of red dwarfs
}

\author{
S. Salimbeni ${ }^{1}$, E. Giallongo ${ }^{1}$, N. Menci ${ }^{1}$, M. Castellano ${ }^{2}$, A. Fontana ${ }^{1}$, A. Grazian ${ }^{1}$, L. Pentericci $^{1}$, D. Trevese ${ }^{2}$, \\ S. Cristiani ${ }^{3}$, M. Nonino ${ }^{3}$, and E. Vanzella ${ }^{3}$ \\ 1 INAF - Osservatorio Astronomico di Roma, via Frascati 33, 00040 Monteporzio (RM), Italy \\ e-mail: salimbeni@mporzio.astro.it \\ 2 Dipartimento di Fisica, Universitá di Roma "La Sapienza", P.le A. Moro 2, 00185 Roma, Italy \\ 3 INAF - Osservatorio Astronomico di Trieste, via G.B. Tiepolo 11, 34131 Trieste, Italy \\ Received 28 May 2007 / Accepted 18 October 2007
}

ABSTRACT

\begin{abstract}
Aims. We study the evolution of the galaxy population up to $z \sim 3$ as a function of its colour properties. In particular, luminosity functions and luminosity densities were derived as a function of redshift for the blue/late and red/early populations.

Methods. We use data from the GOODS-MUSIC catalogue, which have typical magnitude limits $z_{850} \leq 26$ and $K_{\mathrm{s}} \leq 23.5$ for most of the sample. About $8 \%$ of the galaxies have spectroscopic redshifts; the remaining have well calibrated photometric redshifts derived from the extremely wide multi-wavelength coverage in 14 bands (from the $U$ band to the Spitzer $8 \mu \mathrm{m}$ band). We have derived a catalogue of galaxies complete in the rest-frame $B$-band, which has been divided into two subsamples according to their rest-frame $U-V$ colour (or derived specific star formation rate) properties.

Results. We confirm a bimodality in the $U-V$ colour and specific star formation rate of the galaxy sample up to $z \sim 3$. This bimodality is used to compute the luminosity functions of the blue/late and red/early subsamples. The luminosity functions of the blue/late and total samples are well represented by steep Schechter functions evolving in luminosity with increasing redshifts. The volume density of the luminosity functions of the red/early populations decreases with increasing redshift. The shape of the red/early luminosity functions shows an excess of faint red dwarfs with respect to the extrapolation of a flat Schechter function and can be represented by the sum of two Schechter functions. Our model for galaxy formation in the hierarchical clustering scenario, which also includes external feedback due to a diffuse UV background, shows a general broad agreement with the luminosity functions of both populations, the larger discrepancies being present at the faint end for the red population. Hints on the nature of the red dwarf population are given on the basis of their stellar mass and spatial distributions.
\end{abstract}

Key words. galaxies: distances and redshifts - galaxies: evolution - galaxies: high-redshift galaxies: luminosity functions, mass function

\section{Introduction}

The evolution of galaxy luminosity function (LF) is one of the main tools used to study the structure evolution through the cosmic time. The advent of large surveys has allowed the analysis of sub-samples of galaxies selected as a function of their morphological, spectroscopic or colour properties (Strateva et al. 2001; Norberg et al. 2002; Madgwick et al. 2002; Wolf et al. 2003; Willmer et al. 2006; Bell et al. 2004; Baldry et al. 2004; Hogg et al. 2004; Weiner et al. 2005; Blanton et al. 2005; Ilbert et al. 2006; Marchesini et al. 2007; Baldry et al. 2006; Driver et al. 2006; Cucciati et al. 2006; Cirasuolo et al. 2007, Arnouts et al. 2007; Giallongo et al. 2005, elsewhere G05). These studies have allowed us to probe the evolution of galaxies that have different star formation histories.

Of special interest are the studies concerning the statistical properties of galaxies selected on the basis of their intrinsic colour distribution. This distribution appears bimodal up to $z \sim 2-3$ (Baldry et al. 2004; Blanton et al. 2005; Giallongo et al. 2005) and separates the galaxies into two populations, red early types and blue late types. It has been shown that this spectral classification is roughly consistent with the corresponding morphological classification (bulge vs. disk dominated) at least at low and intermediate redshifts (Strateva et al. 2001; Weiner et al. 2005).
This bimodal colour distribution can be naturally explained in hierarchical models for galaxy formation (Menci et al. 2005, 2006) where two distinct populations arise in the colour distribution based on two different star formation histories affected by the feedback effects produced by the supernova (SN) and active galactic nucleus (AGN) activities (Menci et al. 2006). However, the effect of environmental density on the paths of galaxy evolution can have a fundamental role. In this context it is not clear whether the evolutionary history of galaxies is originated by a nurture scenario (galaxy properties are affected by environment through physical mechanisms acting on galaxies) or by a nature scenario (the evolution is driven by the initial condition established during the formation epoch of galaxies, e.g. Mateus et al. 2007; Cooper et al. 2007).

Recent studies have estimated the shape and evolution of the LF of galaxies selected according to their bimodal colour distribution using both the large local Sloan survey (Baldry et al. 2004; Blanton et al. 2005), and other surveys at intermediate and high redshifts (Bell et al. 2003; Giallongo et al. 2005; Faber et al. 2007; Willmer et al. 2006; Ilbert et al. 2006). Their results show the red LF evolving mildly in densities up to $z \sim 1$ with a relatively flat shape at the faint-end, although the evaluation of the faint-end slope of the red LF remains an open issue especially at intermediate and high redshifts where the present surveys do not constrain the faint slope very well (Faber et al. 2007; Bell et al. 2003). 
In G05 we studied the red and blue LFs, using the properties of bimodality in colour and in specific star formation rate (SSFR), with a complete but relatively small sample of galaxies selected in the rest-frame $B$-band from low to high redshifts. We showed that the bimodality extends up to at least $z \sim 2.5$. We also found that the red/early galaxies decrease in their luminosity density by a factor of $\sim 5-6$, from $z \sim 0.5$ to $z \sim 2.5-3$, in broad agreement with the hierarchical cold dark matter model. These results provided a first picture of the evolution of the red and blue LFs up to high redshifts relaying on a relatively deep but small sample. For a more reliable picture a wider sample at high redshift is clearly needed. For this purpose larger areas with deep near-IR imaging are required.

Thanks to the wide area $\left(\sim 140 \operatorname{arcmin}^{2}\right)$ and deep near-IR observations, the GOODS-South survey provides a good starting point for the study of the galaxy properties at high redshift. In particular, the inclusion of the deep IR observations obtained with the Spitzer telescope represent a useful constraint for the estimate of the physical properties of galaxies at high redshift. Last but not least the extensive spectroscopic follow up obtained in this field provides a wide set of spectroscopic redshifts. From this public data set we have obtained a multi-colour catalogue of galaxies, which we have named GOODS-MUSIC (GOODS MUlticolour South Infrared Catalog, Grazian et al. 2006a). This catalogue, where galaxies are selected both in the $z_{850}$ and $K_{\mathrm{s}}$ bands, contains information in 14 bands from the $U$ to the Spitzer $8 \mu \mathrm{m}$ band, and all the available spectroscopic information. For all the objects without spectroscopic information, we have obtained well calibrated photometric redshifts by means of our photometric redshift code (Fontana et al. 2000).

The GOODS-MUSIC catalogue has already been used to investigate the physical and clustering properties of high redshift galaxies (Fontana et al. 2006; Grazian et al. 2006b, 2007; Pentericci et al. 2007; Castellano et al. 2007). Here we study the galaxy LFs of the red and blue populations, enlightening evolutionary features that are characteristic of the two populations.

The paper is organised as follows. In Sect. 2 we describe the basic features of our dataset. In Sect. 3 we describe the bimodality properties of the sample and we define the loci of minimum for the selection of the red/early and the blue/late sub-samples as a function of $z$. In Sect. 4 we compute the shape and evolutionary properties of the LFs and the luminosity density of both populations. Section 5 is devoted to the analysis of the physical properties of the faint early population.

All the magnitudes used in the present paper are in the AB system. An $\Omega_{\Lambda}=0.7, \Omega_{\mathrm{M}}=0.3$, and $H_{0}=70 \mathrm{~km} \mathrm{~s}^{-1} \mathrm{Mpc}^{-1}$ cosmology is adopted.

\section{The GOODS-MUSIC sample}

We use the multicolour catalogue extracted from the southern field of the GOODS survey, located in the Chandra Deep Field South. The procedure adopted to extract the catalogue is described in detail in Grazian et al. (2006a). Here, we summarise the general features.

The photometric catalogue was obtained by combining 14 images from the $U$-band up to $8 \mu \mathrm{m}$. More specifically, it includes two $U$ band images from the ESO $2.2 \mathrm{~m}$ telescope, a $U$ image from VLT-VIMOS, the ACS-HST images in four bands $B, V, I$ and $z_{850}$, the VLT-ISAAC $J, H$ and $K_{\mathrm{s}}$ bands, and the Spitzer bands at 3.6, 4.5, 5.8 and $8 \mu \mathrm{m}$. All the images analysed have an area of $143.2 \mathrm{arcmin}^{2}$, except for the U-VIMOS image (90.2 $\left.\mathrm{arcmin}^{2}\right)$ and the $H$ image $\left(78.0 \mathrm{arcmin}^{2}\right)$. The multicolour catalogue contains 14847 objects, selected either in the $z$ and/or in the $K_{\mathrm{s}}$ band (version 1.0). As in previous papers (Poli et al. 2003; G05) we select galaxies in different bands depending on the redshift interval; more specifically, we select galaxies in the $z$ band at low redshifts $(0.2-1.1)$ and in the $K_{\mathrm{s}}$ band at higher redshifts (1.1-3.5). This allows us, as explained below, to estimate the galaxy LF in the rest frame $4400 \AA$ in the overall redshift interval (0.2-3.5). As stated by Cameron \& Driver (2007) (see also Trujillo et al. 2006), a careful analysis of the selection effects due to the detection completeness is needed. This issue is discussed in the paper by Grazian et al. (2006a). In this paper, we evaluated, using simulations in the $z_{850}$ and $K_{\mathrm{s}}$ bands, a $90 \%$ completeness level for elliptical and spiral galaxies of different half-light radii and bulge/disk ratios. Since the depth of the image used for the galaxy selection varies across the area, a single magnitude limit cannot be defined in each band. As a consequence we divided the $z$-selected sample and the $K_{\mathrm{s}}$-selected sample into six independent catalogues, each with a well defined area and magnitude limit, relative to a $90 \%$ completeness level. The $z$-selected catalogues have magnitude limits in the range 24.65-26.18, while the magnitude limits in the $K_{\mathrm{s}}$-selected sample range from 21.60 to 23.80 , but for most of the sample the typical magnitudes limits are $z_{850} \sim 26.18$ and $K_{\mathrm{s}} \sim 23.5$.

In summary, the $z$-selected sample has 9862 (after removing AGNs and galactic stars) galaxies with about $10 \%$ having spectroscopic redshift, while the $K_{\mathrm{s}}$-selected sample has 2931 galaxies with about $27 \%$ having spectroscopic redshifts. For the galaxies without spectroscopic redshift we use the photometric one. Our photometric redshift technique has been described in Giallongo et al. (1998) and Fontana et al. (2000). We adopt a standard $\chi^{2}$ minimisation over a large set of templates obtained from synthetic spectral models. In particular, we use those obtained with PÉGASE 2.0 (Fioc \& Rocca-Volmerange 1997) as described in Grazian et al. (2006a). The comparison with the spectroscopic subsample shows that the accuracy of the photometric estimation is very good, with $\langle|\Delta z /(1+z)|\rangle=0.045$ in the redshift interval $0<z<6$.

As in Poli et al. (2003) and Giallongo et al. (2005), great care was given to the selection of the sample suited for the estimate of the LF. Indeed, we used the $z$-selected sample for galaxies with $z<1$, where the $4400 \AA$ rest-frame wavelength is within or shorter than the $z_{850}$-band. For this reason we only included in our LF galaxies with $m[4400(1+z)] \leq z_{A B}($ lim $)$. This selection guarantees a completeness of the LF sample at $z<1$ independently of the galaxy colour, although some galaxies from the original $z$-limited sample are excluded since they have a red spectrum, and consequently a magnitude $m[4400(1+z)]$ fainter than our adopted threshold. The same procedure was adopted at higher $(z=1.0-3.5)$ redshifts using the $K_{\mathrm{s}}$-selected sample. The sample selected as described above was adopted for all the analysis presented in this paper.

The method adopted to estimate the rest-frame magnitude and the other physical parameters is described in previous papers (Poli et al. 2003; Giallongo et al. 2005; Fontana et al. 2006). Briefly, it is based on a set of templates, computed with standard spectral synthesis models (Bruzual \& Charlot 2003), chosen to broadly encompass the variety of star-formation histories, metallicities and extinctions of real galaxies. To provide a comparison with previous works, we used the Salpeter IMF, ranging over a set of metallicities (from $Z=0.02 Z_{\odot}$ to $Z=2.5 Z_{\odot}$ ) and dust extinctions $(0<E(B-V)<1.1$, with a Calzetti extinction curve). Details are given in Table 1 of Fontana et al. (2004). For each model of this grid, we computed the expected magnitudes in our filter set, and found the best-fitting template 
with a standard $\chi^{2}$ minimisation, fixing the redshift to the spectroscopic or to the photometric one. The best-fit parameters of the galaxy were found after scaling to the actual luminosity of each observed galaxy. Uncertainties in this procedure produced, on average, small errors $(\leq 10 \%)$ in the rest-frame luminosity (Ellis 1997; Pozzetti et al. 2003). Moreover, the inclusion of the 4 Spitzer bands, longward of $2.2 \mu \mathrm{m}$, for galaxies at $z>2$, was essential to sample the spectral distribution in the rest-frame optical and near-IR bands, and to provide reliable constraints on the stellar mass and dust estimation (for a detailed analysis see Fontana et al. 2006).

\section{The semi-analytical model}

In order to make a comparison with current hierarchical models of galaxy formation we used our semi-analytical model (SAM), described here briefly (for a detailed description see Menci et al. 2005, 2006).

The model connects i) the processes related to the gas physics (emission, radiative cooling, heating); ii) the star formation activity (whose rate is assumed to proceed from the conversion of the cold gas mass on a timescale proportional to the disk dynamical timescale); and iii) the consequent Supernovae heating of the gas to the merging histories of dark matter haloes. The model also includes the effect of starbursts triggered by galaxy interactions and the accretion onto supermassive black holes at the centre of galaxies powering the AGN activity, with the corresponding energy feedback onto the interstellar medium.

We adopt the same choice for the model free parameters (the normalisation of the star formation timescale and of the Supernovae energy feedback) as in the above papers; the only changes concern the use of merger trees with a larger mass resolution $\left(M=10^{9} M_{\odot}\right)$ for progenitors of large mass $(M>$ $10^{14} M_{\odot}$ ) haloes, and the complete depletion of gas in haloes with a virial temperature lower than $4 \times 10^{4} \mathrm{~K}$, due to the effect of the UV background (see also Somerville \& Primack 1999).

\section{Bimodality}

\subsection{Colour and SSFR properties of the GOODS-MUSIC sample}

The recent analysis of the spectral properties of galaxies selected in large or deep surveys has shown the presence of a strong bimodality in their colour distribution (e.g. Strateva et al. 2001; Baldry et al. 2004; Willmer et al. 2006), allowing the identification of two main populations, red/early and blue/late galaxies, mainly on the basis of a single colour, e.g. the rest-frame $U-V$. The local distribution has been studied by Strateva et al. (2001) and Baldry et al. (2004) in the framework of the Sloan survey and, at intermediate and high redshifts, by several authors (Bell et al. 2004; Giallongo et al. 2005; Weiner et al. 2005; Cirasuolo et al. 2007; Cucciati et al. 2006; Franceschini et al. 2006). Some effort has been devoted to explaining the observed bimodality in the framework of the hierarchical clustering picture (Menci et al. 2005, 2006; Dekel \& Birnboim 2006). In particular, Menci et al. (2005) proposed that the colour bimodality arises because of two natural features: the star formation histories of the massive red galaxies, which are formed in biased high-density regions, and are peaked at higher $z$ as compared to lower mass galaxies; and the existence of a non-gravitational mass scale $\left(m_{0}\right)$. For $m<m_{0}$ the star formation is self regulated and the cold gas content is continuously depleted by SN feedback, for $m>m_{0}$ the cold gas is not effectively reheated and so the rapid cooling takes place at high- $z$. These different evolutionary paths led to the present day red and blue populations (Menci et al. 2005). When the energy injection from AGN feedback is included (Menci et al. 2006), the bimodal distribution appears at even higher redshifts $(z>2)$.

Using the rest-frame colour we can separate the red population from the blue to analyse the evolution of the LFs. A recent analysis of the morphological structure of a fraction of the GOODS sample has shown a good correlation between the red colour and the spheroidal morphology of galaxies up to $z \sim 1.5$ (see Franceschini et al. 2006). Moreover, as in G05, we are interested in the galaxy evolution as a function of the star-formation activity. In this respect, the use of the colour criterion introduces some population mixing for the red galaxies since it is not possible to distinguish an early-type galaxy from a dusty star-burst using only the rest-frame $U-V$ colour. Therefore, we used the Bruzual \& Charlot (2003) spectral best fit of the individual galaxy spectral energy distributions (SEDs) to derive the SSFR, $\dot{m}_{*} / m_{*}$ (as described in the previous section). We are aware that the absolute values in the $\dot{m}_{*} / m_{*}$ distribution are subject to uncertainties due, for example, to the estimate of the dust attenuation, which depends on the extinction curve adopted, and to the methods adopted for the mass estimate. We refer to our previous paper, G05 and references therein, for a description of the method used and its reliability. However, although some degeneracy still remains, we can use this property to separate our sample, removing the obvious star-burst galaxies from the locus of early-type.

The results about the colour bimodality at high redshift from G05 are here confirmed at a higher statistical level. The colourluminosity relation is shown in Fig. 1, while the analogous distribution in SSFR is shown in Fig. 2.

The minima in the colour-magnitude distribution and in the SSFR-magnitude distribution are used to divide the sample into red/early and blue/late populations. For an evaluation of this relation we adopted the same procedure as in G05. We fit the distributions shown in Figs. 1 and 2 with the sum of two Gaussians whose mean is a linear function of the absolute magnitude $M_{B}$. Each Gaussian has a constant dispersion and each sub-sample of galaxies, with a different magnitude limit, has been weighted with its covering sky area. Since the statistics of the red population are still poor, we adopted, as in G05, the same dependence on the absolute magnitude for both the blue and the red populations. Taking into account the different normalisations of the two Gaussian distributions we then derived the locus of the formal minimum in the sum of the two Gaussians, separating in this way the two populations. The loci of the red/early and blue/late populations are shown in Table 1, and the minima in Table 2.

The resulting colour distribution projected at $M_{B}=-20$ along the best-fit correlation is also displayed in Fig. 1, with the vertical line indicating the colour separation at that magnitude.

The same is shown for the SSFR distribution in Fig. 2 where the relative numbers of early and late type galaxies can also be derived in two different ranges of absolute magnitudes.

The uncertainty associated with the selection of the minima has been derived reproducing 100 colour-magnitude plots with a MonteCarlo analysis using 100 galaxy catalogues. In each catalogue we assigned to each object a different redshift drawn from its probability distribution and we associated their rest-frame absolute magnitudes and SSFRs. The $z$ probability distribution naturally takes into account the photometric errors and the model degeneracy in the spectral libraries. The uncertainty region is shown in Figs. 1 and 2 as a shaded area, its value is $\leq 0.1$ for the minima in colour, and $\sim 0.2$ for those in SSFR. 


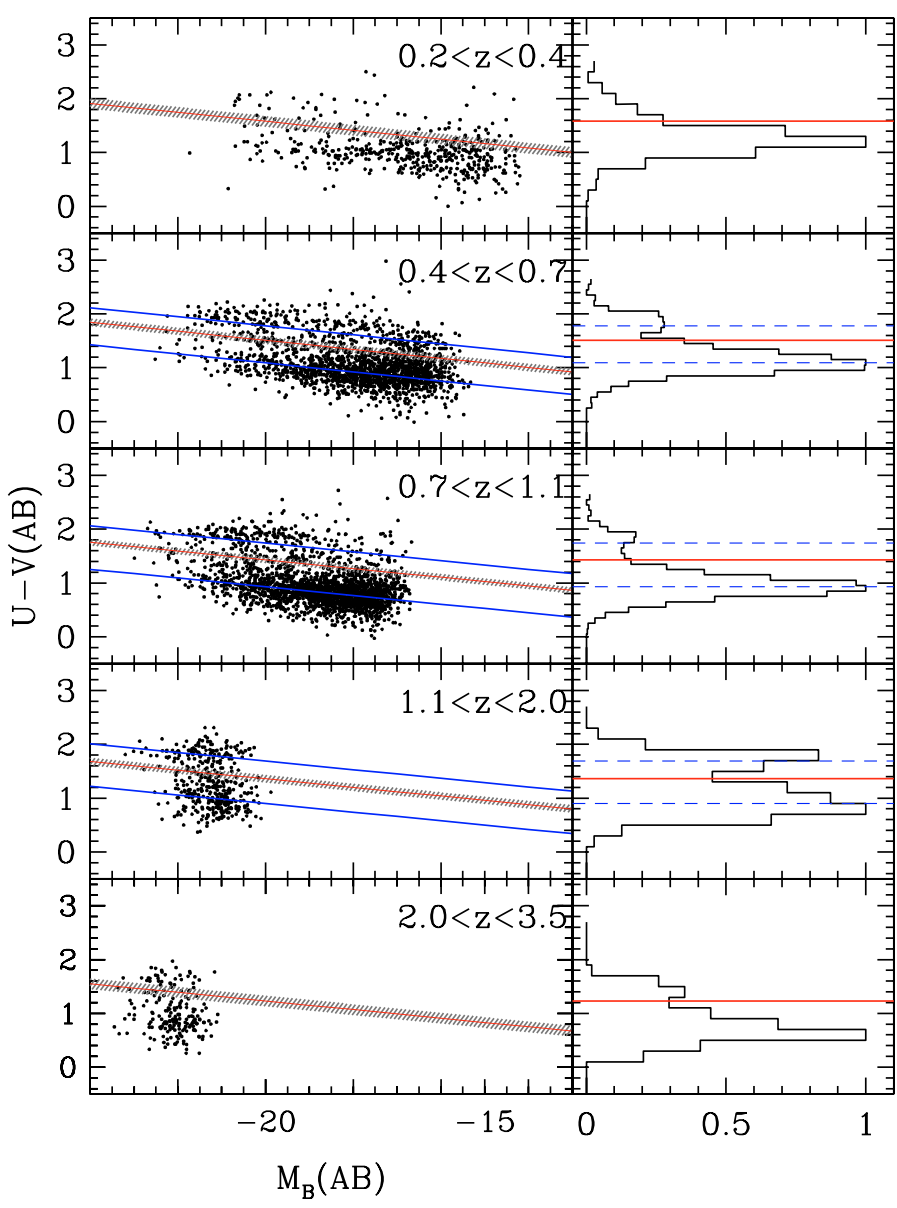

Fig. 1. Left panel: colour-magnitude diagrams in various redshift intervals; the lines represent the best-fit relations for the blue and red populations and the locus of the minimum, the shaded area is the uncertainty on the minimum. Right panel: the histograms of colour distribution projected at $M_{B}=-20$ along the best-fit correlations, the continuous horizontal lines are the colour separation at this magnitude, and the dash horizontal lines are the loci of the maxima.

The colour/SSFR-magnitude relations for the loci of the maxima and minima follow the linear relations $\langle U-V\rangle=$ $\alpha\left(M_{B}+20\right)+\langle U-V\rangle_{20}$ and $\langle S S F R\rangle=\alpha\left(M_{B}+20\right)+\langle S S F R\rangle_{20}$, whose parameters are listed in Tables 1 and 2 .

In the colour-magnitude relation we confirm the weak intrinsic blueing with increasing redshift from $z \sim 0.4$ to $z \sim 2$ already found by G05 for both populations, although formally only at $2 \sigma$ level. In the redshift bins where the statistics are poor, the minimum is extrapolated from the other redshift bins. From the bins $z=0.4-0.7$ and $z=0.7-1.0$ we extrapolate the minimum value $\langle U-V\rangle_{20}=1.58$ in the redshift interval $z=0.2-0.4$, and from the bins $z=0.7-1.0$ and $z=1.0-2.0$ we extrapolate $\langle U-V\rangle_{20}=1.23$ in the interval $z=2-3.5$.

We found no appreciable redshift evolution in the SSFR distribution, so in order to increase the statistics we performed the fit in the larger redshift intervals $z=0.2-1$ and $1-3.5$. Moreover, there is no appreciable dependence of SSFR on the absolute magnitude, so the colour-magnitude relation is not related to similar trends in the SSFR.

\subsection{Discussion on the selection criteria}

One notes in the colour/SSFR-magnitude relations the presence of a conspicuous number of intrinsically faint galaxies with

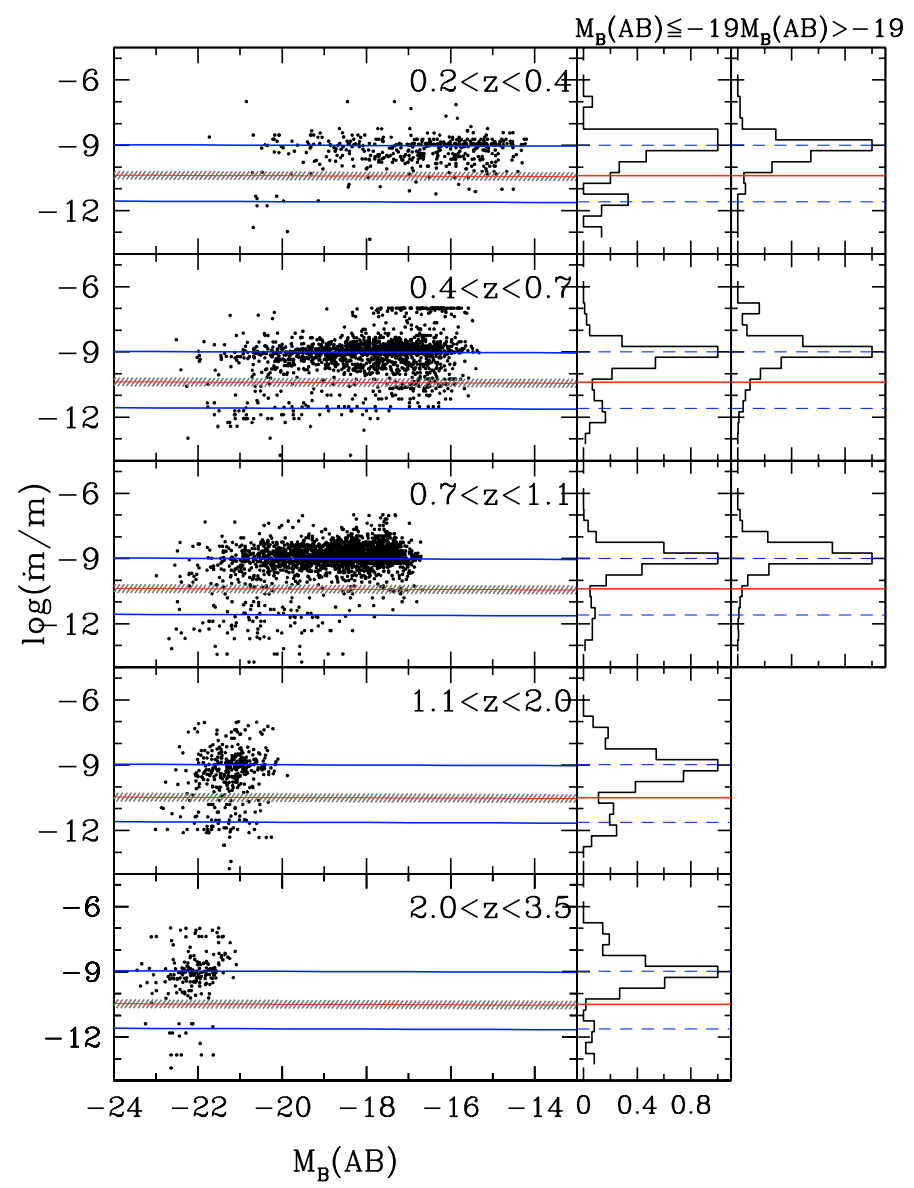

Fig. 2. As in Fig. 1 but for the SSFR-magnitude distribution.

relatively red colours. They are red with respect to the locus of separation of the two populations although, because of the colour-magnitude relation, their colours are typical of the bright $\left(M_{B} \sim-22\right)$ blue galaxies. In terms of SSFR these galaxies show intermediate values between star forming and early type galaxies. The presence of a large number of galaxies belonging to this intermediate population dominates the shape of the LF of the red/early type galaxies at the faint end, as shown in the next sections.

However, since the colour dispersion of the blue sequence broadens at faint magnitudes, the assumption of a linear parameterisation for the minimum could imply a bias for the selection of the red sample (for a detailed analysis of the blue sequence properties see Labbé et al. 2007). For this reason we also performed an analysis deriving the minimum without any assumption on its dependence on the rest-frame luminosity. We concentrated our analysis in the redshift interval 0.4-0.7, which has sufficient statistics at faint magnitudes. We obtained a volume corrected colour distribution in four magnitude bins, and for each colour distribution we fit a double-Gaussian function, as shown in Fig. 3. We adopted the intersection of the two Gaussians as minimum. In this way we verified that the locus of the minimum is well described by a linear behaviour: by performing a linear fit to these points we found the following relation $\langle U-V\rangle=-0.07 \cdot\left(M_{B}+20\right)+1.57$ (see Fig. 4). This relation is very similar to our standard analysis and does not produce a substantial variation of our results (see also Sect. 5.3 and Fig. 8). As described above, this analysis can not exclude a contamination from the star-burst galaxies reddened by dust, for this reason we 
Table 1. Parameters of the relation between the loci of the maxima and the absolute $B$ magnitude.

\begin{tabular}{cccr}
\hline \hline$\langle z\rangle$ & $\alpha$ & $m_{\text {red/early }}$ & $m_{\text {blue } / \text { late }}$ \\
\hline \multicolumn{4}{c}{$U-V$} \\
\hline 0.40 .7 & $-0.08 \pm 0.01$ & $1.79 \pm 0.05$ & $1.09 \pm 0.03$ \\
0.71 .1 & $-0.08 \pm 0.01$ & $1.74 \pm 0.05$ & $0.93 \pm 0.03$ \\
1.12 .0 & -0.08 & $1.69 \pm 0.05$ & $0.90 \pm 0.03$ \\
\hline \multicolumn{4}{c}{ SSFR } \\
\hline 0.21 .1 & $0.019 \pm 0.03$ & $-11.59 \pm 0.20$ & $-9 . \pm 0.03$ \\
1.13 .5 & 0.019 & $-11.55 \pm 0.20$ & $-9.07 \pm 0.03$ \\
\hline
\end{tabular}

Table 2. Parameters of the relation between the locus of the minimum and the absolute $B$ magnitude.

\begin{tabular}{lcc}
\hline \hline$\langle z\rangle$ & $\alpha$ & minimum \\
\hline \multicolumn{3}{c}{$U-V$} \\
\hline 0.20 .4 & -0.08 & $1.58 \pm 0.10^{*}$ \\
0.40 .7 & $-0.08 \pm 0.01$ & $1.51 \pm 0.07$ \\
0.71 .1 & $-0.08 \pm 0.01$ & $1.43 \pm 0.06$ \\
1.12 .0 & -0.08 & $1.36 \pm 0.07$ \\
2.03 .5 & -0.08 & $1.23 \pm 0.10^{*}$ \\
\hline \multicolumn{3}{c}{ SSFR } \\
\hline 0.21 .1 & $0.019 \pm 0.020$ & $-10.41 \pm 0.20$ \\
1.13 .5 & 0.019 & $-10.43 \pm 0.20$ \\
\hline
\end{tabular}

${ }^{*}$ Extrapolated value.

also performed an analysis of the LF on a homogeneous sample of galaxies selected using SSFR distribution.

\section{Luminosity function}

\subsection{The statistical analysis}

The LF is computed with the procedure described in G05. We applied to our sample an extended version of the standard $1 / V_{\max }$ algorithm (Avni \& Bahcall 1980). As in the previous paper, we used a combination of data derived from regions in the field with different magnitude limits. Indeed, for each object and for each $j$ th region under analysis a set of effective volumes $V_{\max }(j)$ is computed. For a given redshift interval $\left(z_{1}, z_{2}\right)$, these volumes are enclosed between $z_{1}$ and $z_{\text {up }}(j)$, the latter being defined as the minimum between $z_{2}$ and the maximum redshift at which the object could have been observed, within the magnitude limit of the $j$ th field. The galaxy number density $\phi(M, z)$ in each $(\Delta z, \Delta M)$ bin can then be obtained as:

$\phi(M, z)=\frac{1}{\Delta M} \sum_{i=1}^{n}\left[\sum_{j} \omega(j) \int_{z_{1}}^{z_{\mathrm{up}}(i, j)} \frac{\mathrm{d} V}{\mathrm{~d} z} \mathrm{~d} z\right]^{-1}$

where $\omega(j)$ is the area in units of steradians corresponding to the field $j, n$ is the number of objects in the chosen bin and $\mathrm{d} V / \mathrm{d} z$ is the comoving volume element per steradian.

The Poisson error in each LF magnitude bin was computed adopting the recipe by Gehrels (1986), valid also for small numbers. The uncertainties in the LF due to the photometric uncertainties and to the degeneracy of the spectral models used to
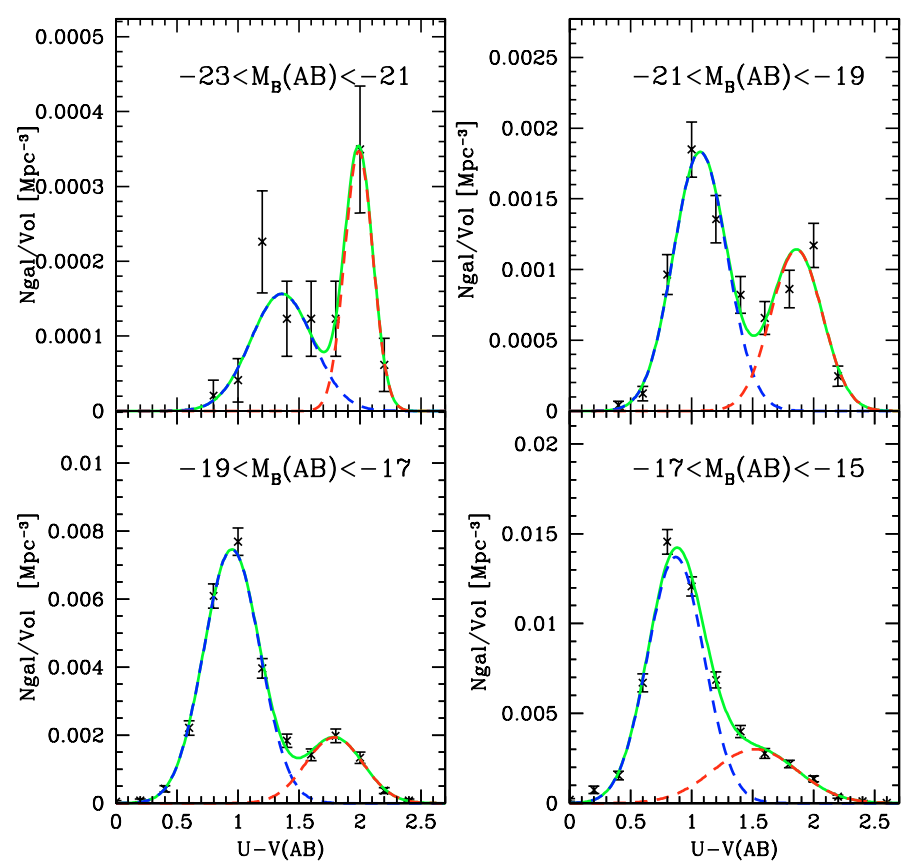

Fig. 3. Volume corrected colour histogram, in four bins of magnitude, in the redshift interval $0.4<z<0.7$. The continuous lines are the double-Gaussian fit to the colour distributions.

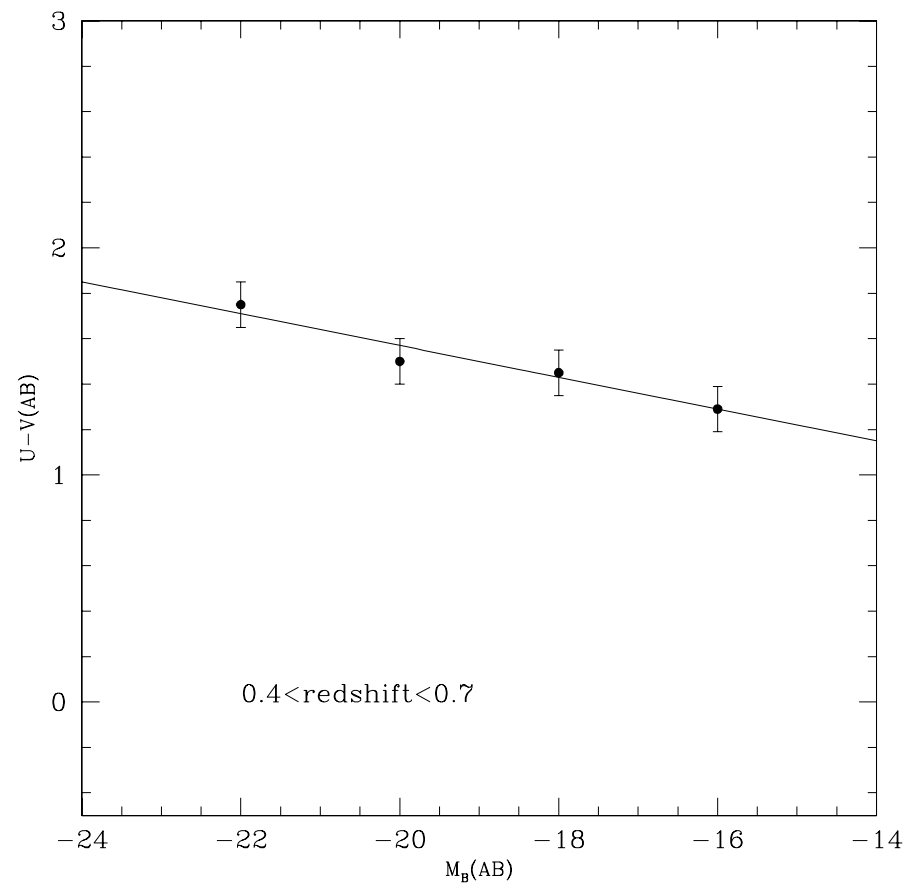

Fig. 4. Points are the minima obtain by the fits shown in Fig. 3. Continuous line is the linear fit to these minima.

derive redshifts were computed with the same Monte Carlo analysis described in the previous section. The uncertainties obtained and the Poisson errors were added in quadrature.

The $1 / V_{\max }$ estimator for the LF can in principle be affected by small scale galaxy clustering. For this reason a parametric maximum likelihood (ML) estimator is also adopted, which is known to be less biased with respect to small scale clustering (see Heyl et al. 1997).

The parametric analysis of the galaxy LF was obtained from the maximum likelihood analysis assuming for different galaxy 
Table 3. LF parameters for the total and blue/late. type sample.

\begin{tabular}{cccccccc}
\hline \hline Type & $z$ & $M_{0}^{*}$ & $\alpha$ & $M_{1}^{*}$ & $z_{\text {cut }}$ & $\phi_{0}^{*}$ & $N$ \\
\hline all & $0.2-3.5$ & $-20.54 \pm 0.12$ & $-1.34 \pm 0.01$ & $0.93 \pm 0.13$ & $0.96 \pm 0.02$ & 0.0031 & 5115 \\
\hline blue & $0.2-3.5$ & $-19.97 \pm 0.20$ & $-1.39 \pm 0.02$ & $1.43 \pm 0.27$ & $0.98 \pm 0.05$ & 0.0024 & 4127 \\
late & $0.2-3.5$ & $-20.06 \pm 0.12$ & $-1.36 \pm 0.02$ & $1.29 \pm 0.12$ & $0.99 \pm 0.02$ & 0.0029 & 4612 \\
\hline
\end{tabular}

populations different parameterisation $\phi$ for the LF. The maximum likelihood method used here represents an extension of the standard Sandage et al. (1979) method, where several samples can be jointly analysed and where the LF is allowed to vary with redshift. A more detailed description can be found in G05 and a formal derivation of the maximum likelihood equation is shown in Heyl et al. (1997).

To describe the $B$-band LF of the total sample and that of blue/late galaxies we assume a Schechter parameterisation:

$$
\begin{aligned}
\phi(M, z)= & 0.4 \ln (10) \phi^{*}\left[10^{0.4\left(M^{*}-M\right)}\right]^{1+\alpha} \\
& \times \exp \left[-10^{0.4\left(M^{*}-M\right)}\right] .
\end{aligned}
$$

Since previous analyses have shown that the evolution of the global galaxy LF is manly driven by luminosity evolution, we adopted an $M^{*}$ that is redshift dependent (see aslo Heyl et al. 1997; Giallongo et al. 2005; Gabasch et al. 2006). We used the parameterisation that better matches our data in this range of redshifts:

$M^{*}(z)=\left\{\begin{array}{l}M_{0}-M_{1} z \text { if } z \leq z_{\text {cut }} \\ M_{0}-M_{1} z_{\text {cut }} \text { if } z>z_{\text {cut }} .\end{array}\right.$

The slope $\alpha$ is kept constant with redshift since it is well constrained only at low-intermediate redshift $(z<1)$. For all of the sample considered here, we checked that the density parameter $\phi$ had no significative variation if it had been evolved with the parametric form described in G05, thus we keep it as constant.

As shown in Figs. 7 and 9 this is not a good description for the red/early population for which we assume a double Schechter form, as frequently adopted in similar cases in literature (e.g. Popesso et al. 2006):

$$
\begin{aligned}
\phi(M)= & \phi_{f}(M)+\phi_{b}(M) \\
= & \phi^{*} 0.4 \ln (10) \\
& \times\left(\left[10^{0.4\left(M_{f}^{*}-M\right)}\right]^{1+\alpha_{f}} \exp \left[-10^{0.4\left(M_{f}^{*}-M\right)}\right]\right) \\
& +\left(\left[10^{0.4\left(M_{b}^{*}-M\right)}\right]^{1+\alpha_{b}} \exp \left[-10^{0.4\left(M_{b}^{*}-M\right)}\right]\right) .
\end{aligned}
$$

For a quantitative evaluation of the density evolution at the bright end, we evolved the bright Schechter with a redshift dependent normalisation:

$\phi(M, z)=\phi_{f}(M)+\operatorname{norm}(z) \phi_{b}(M)$

where

$\operatorname{norm}(z)=\left\{\begin{array}{l}1 \text { if } z<z_{\text {cut }} \\ z^{\gamma}\left(z_{\text {cut }}\right)^{-\gamma} \text { if } z \geq z_{\text {cut }} .\end{array}\right.$

We used the MINUIT package of the CERN library (James \& Roos 1995) for the minimisation. The errors in Tables 3 and 4 are calculated for each parameter, independently of the others.
Table 4. LF parameters for red and early. type galaxies, in the redshift interval $0.4-3.5$.

\begin{tabular}{ccc}
\hline \hline Parameter & Red & Early \\
\hline$N$ & 913 & 472 \\
$\phi_{0}^{*}$ & 0.0020 & 0.0017 \\
$\gamma$ & $-1.045 \pm 0.13$ & $-1.45 \pm 0.16$ \\
$z_{\text {cut }}$ & $0.65 \pm 0.01$ & $0.67 \pm 0.01$ \\
$\alpha_{b}$ & $-0.76 \pm 0.06$ & $-0.40 \pm 0.1$ \\
$M_{b}^{*}$ & $-21.29 \pm 0.1$ & $-21.22 \pm 0.12$ \\
$\alpha_{f}$ & $-1.77 \pm 0.2$ & $-1.54 \pm 0.22$ \\
$M_{f}^{*}$ & $-17.04 \pm 0.12$ & $-17.06 \pm 0.12$ \\
\hline
\end{tabular}

\section{2. $B$-band luminosity function from $z \sim 0.2$ to $z \sim 3.5$ and the comparison with the other surveys}

The evolution of the total LF is shown in Fig. 5. To compare our high $z$ results and our fits with local values we also show the local LF from the Two-Degree Field Galaxy Redshift Survey (2dFGRS; Norberg et al. 2002, dotted line in Fig. 5).

The $1 / V_{\max }$ analysis shows that the main kind of evolution is due to a brightening of the LF with redshift. We applied the ML analysis to the sample using the evolutionary form of the Schechter LF described in Eq. (2), where pure luminosity evolution is allowed up to a maximum redshift beyond which the LF keeps constant. The results in Table 3 imply that the LF is subject to a mild luminosity evolution only up to $z \sim 1\left(\Delta M^{*} \sim 0.7\right.$ in the $z=0.2-1$ interval). At higher $z$ the LF appears constant with redshift, although at $z \sim 3$, in the brightest bin, a slight excess is present. In any case, the adopted evolutionary model is acceptable at $2 \sigma$ level using the standard $\chi^{2}$ test.

We also show a comparison with the DEEP2 Redshift Survey (D2RS, Davis et al. 2003; Willmer et al. 2006, empty points in Fig. 5). Although the comparison of the LFs was performed on data taken from surveys that have different magnitude limits and redshift estimates (photometric and spectroscopic), the agreement is very good in the overlapping magnitude regions and in all the redshift bins. A general good agreement is also found with the LF derived from the VVDS survey by Ilbert et al. (2005), although from our ML analysis we do not have any evidence of steepening of the faint end slope up to $z \sim 2$, as suggested by them. We compared our results with other photometric redshift surveys, like the COMBO-17 survey by Bell et al. (2004) (see also Faber et al. 2007). A good agreement is found in the overall redshift interval and in the appropriate magnitude interval. The comparison with the FORS Deep Field (FDF) (Gabasch et al. 2004) is less straightforward, because of the different redshift intervals used. An acceptable agreement is found up to $z \sim 1$. At higher redshifts the FDF luminosity function shows an excess of very bright objects with respect to our values, as well as the COMBO17 and DEEP2 LFs.

In Fig. 5 we included the LFs derived from our hierarchical model described in Sect. 3 (see also Menci et al. 2005, 2006). 


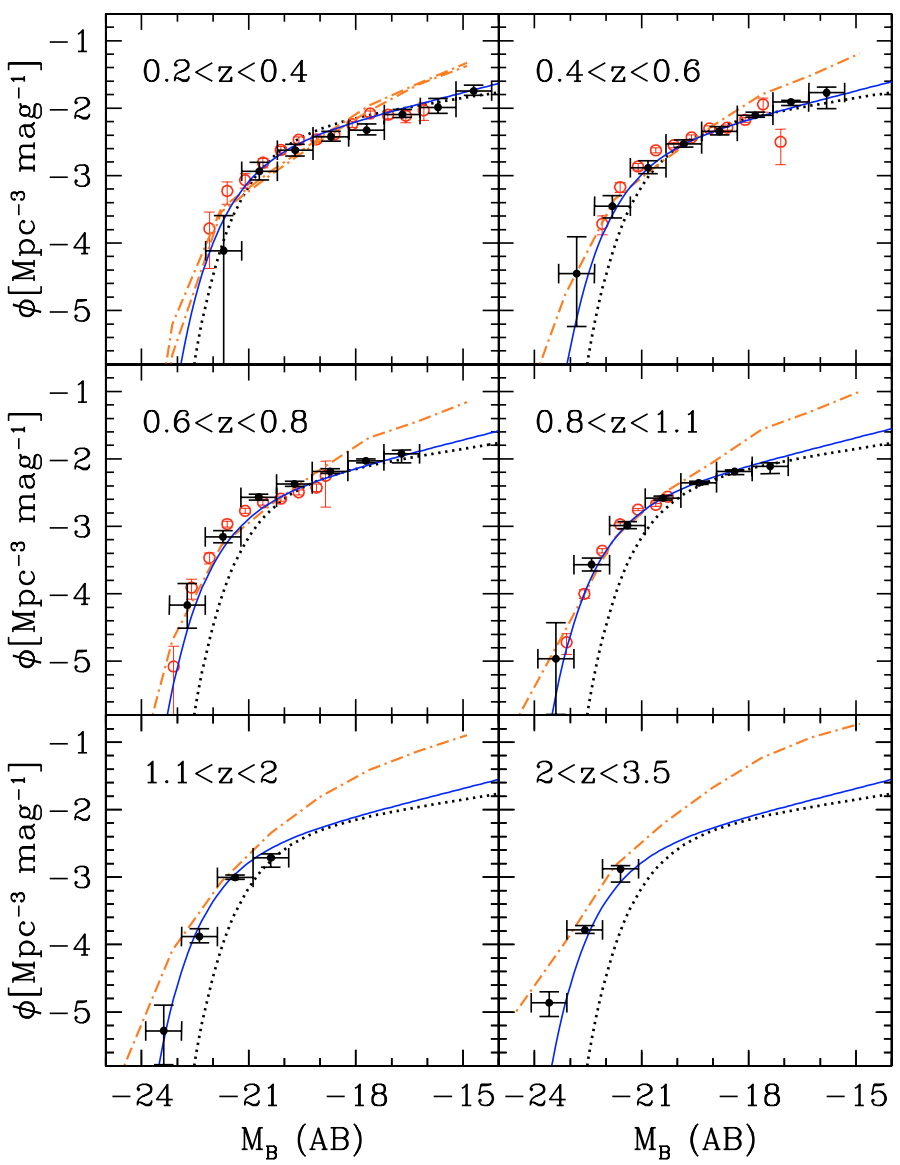

Fig. 5. Total LF as a function of redshift. The continuous curves come from our maximum likelihood analysis. The dotted line is the local LF (Norberg et al. 2002). The filled circles are the points obtained with $1 / V_{M A X}$ method. The empty circles come from the DEEP2 survey (Willmer et al. 2006). The results from the COMBO17 and VDDS surveys are consistent with the DEEP2 results and are omitted from the plot. The dashed-point line is the model of Menci et al. (2006).

The effect of the UV background is effective in flattening the predicted shape of the LF at the faint magnitudes providing an agreement better than that previously obtained (Poli et al. 2003).

\subsection{LF for the blue/late and red/early galaxies}

In this section we show the shapes and evolutionary behaviours of the LFs derived for the blue/late and red/early galaxy populations, respectively. We have adopted the empirical colour/SSFR selection, described in Sect. 4, to separate the two populations.

The shape and redshift evolution of the blue LF is shown in Fig. 6, where both the $1 / V_{\max }$ data points and the curves derived from the ML analysis are represented. The best fit parameters together with their uncertainties are shown in Table 3. The LFs of the late populations are very similar to the blue ones and are not shown in a figure.

As for the total sample, we found that the blue population is well represented by the same type of luminosity evolution, although faster, with $\Delta M^{*} \sim 1.15$ in the $z=0.2-1$ interval. The faint end slope appears steeper. This is due, of course, to the fact that the blue population dominates the volume density of the total sample at any redshift.

We first compare our results with those of the spectroscopic survey D2RS (Faber et al. 2007; Willmer et al. 2006). We note that the colour selection they use to separate the two populations

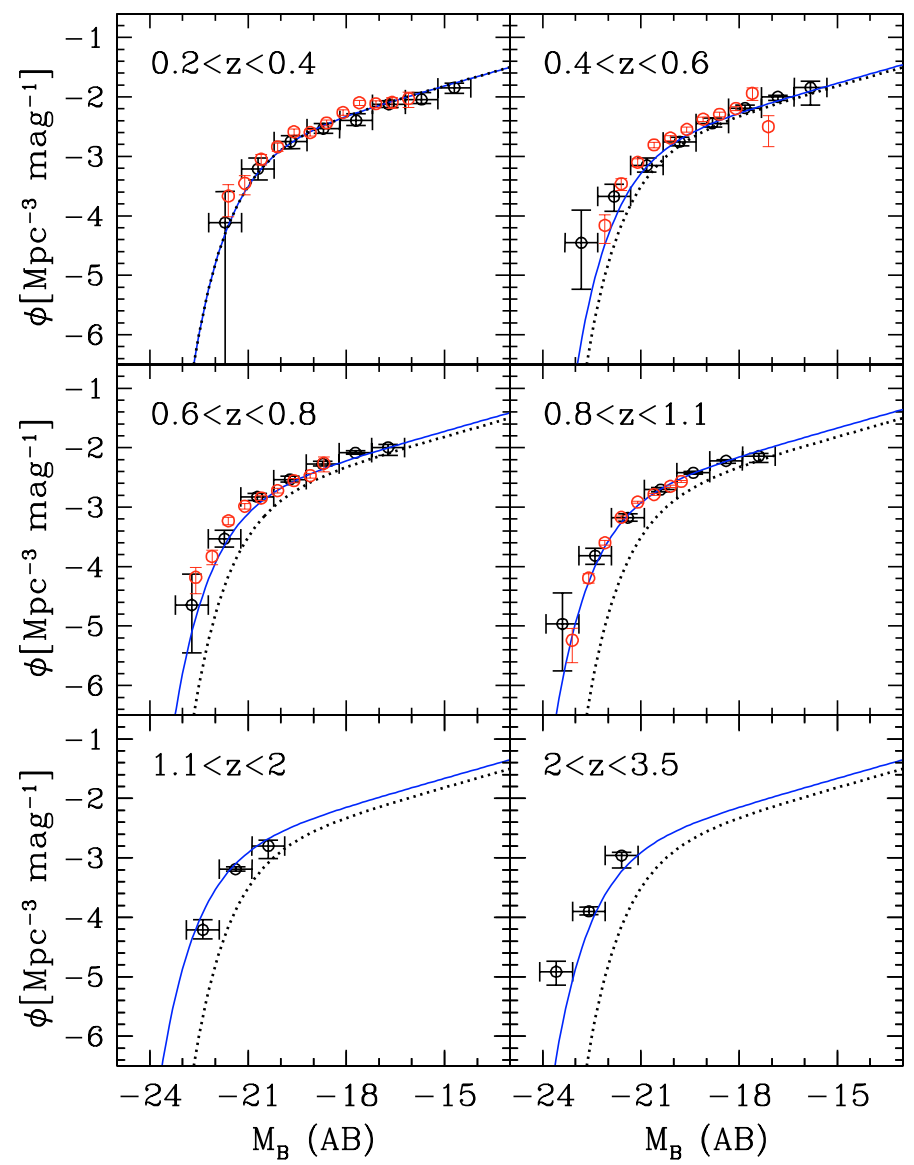

Fig. 6. LFs of the blue galaxies as function of redshift. The continuous curves comes from our maximum likelihood analysis. The dotted line is our fit at $z \sim 0.3$, reported for comparison in all the redshift bins. The big filled circles are the points obtained with $1 / V_{M A X}$ method. The small empty circles are points from the LFs by Willmer et al. (2006).

is based on a $U-B$ vs. $M_{V}$ colour-magnitude relation. We have verified in our sample that their colour selection is very similar to our criterion. In fact, if we adopt their selection on our sample, we reproduce almost the same blue/red galaxy subsamples obtained with our cut. Their LFs are in good agreement with our results, as shown in Fig. 6 (data with error bars). We then compare our results to those of COMBO-17. They use as a selection criterion the colour $U-V$ vs. $M_{V}$, which nearly corresponds to that used by Willmer et al. (2006). The agreement with our results is good.

A direct comparison with the blue/red LFs by Marchesini et al. (2007) in the redshift interval $2<z<3.5$ is not possible since they use a colour separation that is bluer than our criterion by 0.2 mag providing a LF with a lower normalisation.

The LF predicted by our hierarchical model was not included in Fig. 6 since it is not appreciably different from that of the total sample.

Concerning the red/early populations, our GOODS-MUSIC sample allows a sampling of the red LF down to $M \sim-16$ up to $z \sim 0.8$, providing for the first time a direct evaluation of the faint shape of the LF. This is due to the deeper $K$ magnitude limit with respect to that used in $\mathrm{G} 05$, allowing the evaluation of the rest frame $U-V$ colour at magnitudes as faint as $M_{B} \sim-16$. At variance with previous works that involve shallower samples, a peculiar LF shape is present at $z<0.8$ with a minimum and a clear upturn at $M_{B} \geq-18$ (Fig. 7). 


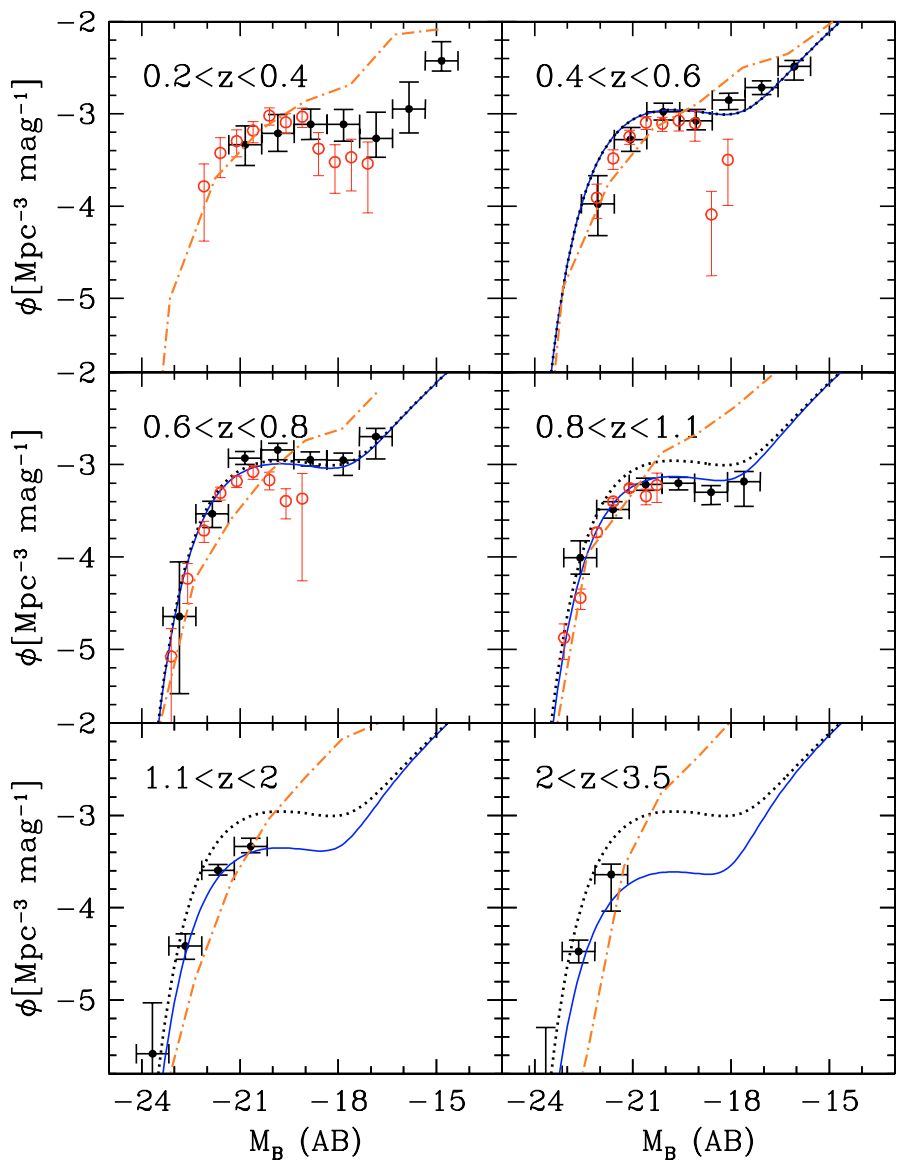

Fig. 7. LFs of the red galaxies as function of redshift. The continuous curves comes from our maximum likelihood analysis. The first bin of redshift, has been excluded from this evolutive analysis since the statistics are too low. The dotted line is our fit at $z \sim 0.5$, reported for comparison in all the redshift bins. The big filled circles are the points obtained with $1 / V_{M A X}$ method. The small empty circles are points from the LFs by Willmer et al. (2006). The dashed-point orange line is the model of Menci et al. (2006).

This overabundance of faint objects, with respect to the extrapolation of the Schechter function, was already found in the LF of local early type galaxies derived from the $2 \mathrm{dF}$ survey (Madgwick et al. 2002). Although they used a different separation criterion, their subsample called type 1 is not so different from our subsample, being equivalent to a morphological sample of E, SO and Sa. A similar upturn was also found in the local LF of red galaxies derived from the Sloan survey by Blanton et al. (2005).

We checked that the characteristic shape found is not critically dependent on the specific choice of the colour-magnitude or SSFR -magnitude separation. Indeed, changing the parameters of the linear fit in colour-SSFR separation at $2-\sigma$ level, the shape and, in particular, the upturn, do not change appreciably. We also found that the overall shape of the LF does not change if we use a separation between blue and red galaxies found with an analysis that has no assumption regarding the parametric dependence on the rest-frame magnitude (see Sect. 4.2). Moreover, when the bimodal distribution in the faintest magnitude interval is fit with a double Gaussian function (see Fig. 3, $-17<M B<-15$ ), the contamination by any blue population in the locus of the red population is not so strong. In other words the FWHM of the blue Gaussian distribution is relatively narrow. In this case, the expected fraction of blue galaxies expected

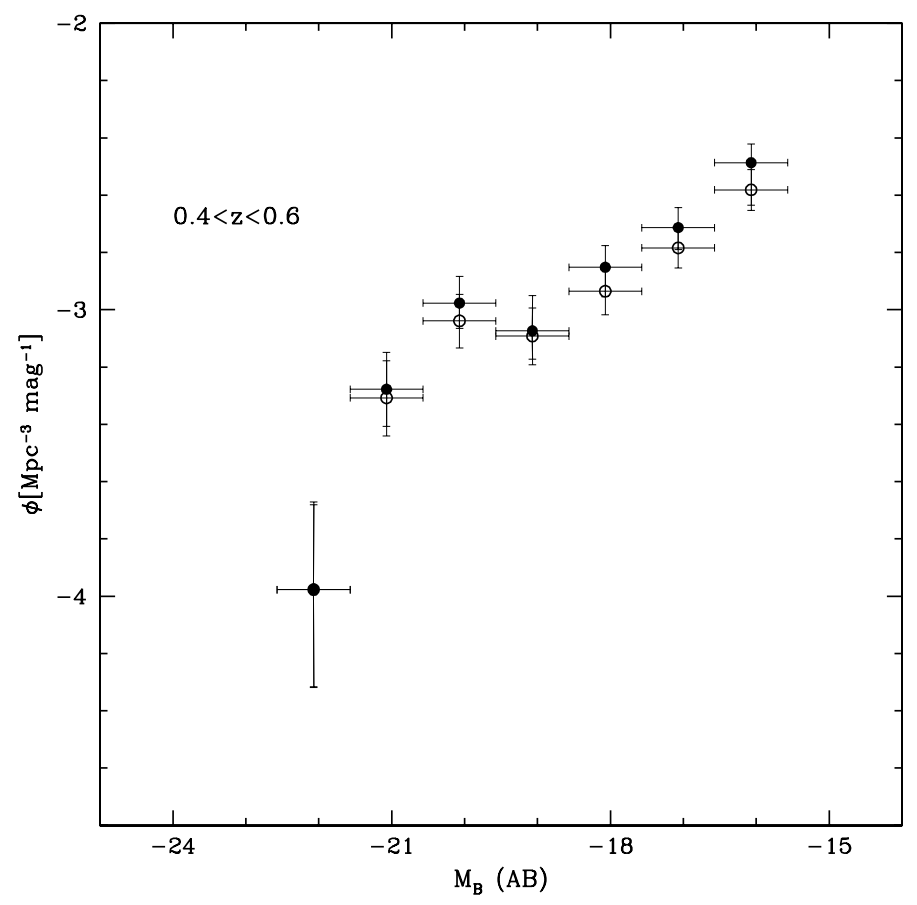

Fig. 8. LFs of the red galaxies. The filled circles indicate the LF obtained with the linear colour selection in Table 2 . The empty circles indicate the LF obtained from the colour histograms in bins of magnitude (see Figs. 3 and 4).

redward of the selected minimum is only $14 \%$ of the red population in the same colour region. For this reason the shape of the red LF shown in Fig. 8 relative to the redshift interval $0.4<z<0.6$ remains almost unchanged in the two fainter magnitude bins if we remove this small fraction.

Also for the red population we compared our LFs with those derived from the major surveys of colour selected galaxies. In Fig. 7 we show the LF from the spectroscopic survey of Willmer et al. (2006). For the red galaxies the agreement is good for $M_{B}<-20$, where the incompleteness is negligible in their sample (see Fig. 8, Willmer et al. 2006). Their shallower sample cannot probe the raise at the faint end present in our deeper data. The same holds for the two photometric surveys of Bell et al. (2004) and Brown et al. (2007).

Concerning the parametric analysis of the evolutionary LFs of the red/early galaxy population, given the excess of faint objects, a single Schechter shape does not provide an acceptable description of the data. For this reason we adopted a double Schechter function, as described in Eq. (4). The best fit parameters are shown in Table 4 . The best fit value of the brighter Schechter slope is rather flat $\left(\alpha_{b} \sim-0.7\right)$, in agreement with what was found in G05 and in the Bell et al. sample. The fainter slope is steeper, approaching the value $\alpha_{f} \sim-1.8$. As for the redshift evolution we adopted the density evolution law described in Eq. (5) where the Schechter shape at the faint end is kept constant at all redshifts. The brighter one is constant only up to a given redshift $z_{\text {cut }}$, beyond which it decreases as a power law in redshift. We find a constant density up to $z \sim 0.7$ and thereafter a decrease by a factor of $\sim 5$ up to $z \sim 3.5$.

The LF evolution of the early galaxies selected from their SSFR value is very similar to that of the red ones, although the high redshift density evolution is more pronounced with a decrease by a factor of $\sim 10$ in the interval $z=0.7-3.5$. This difference is caused by the presence, in the red sample at higher redshift, of a high fraction of galaxies having SEDs consistent 


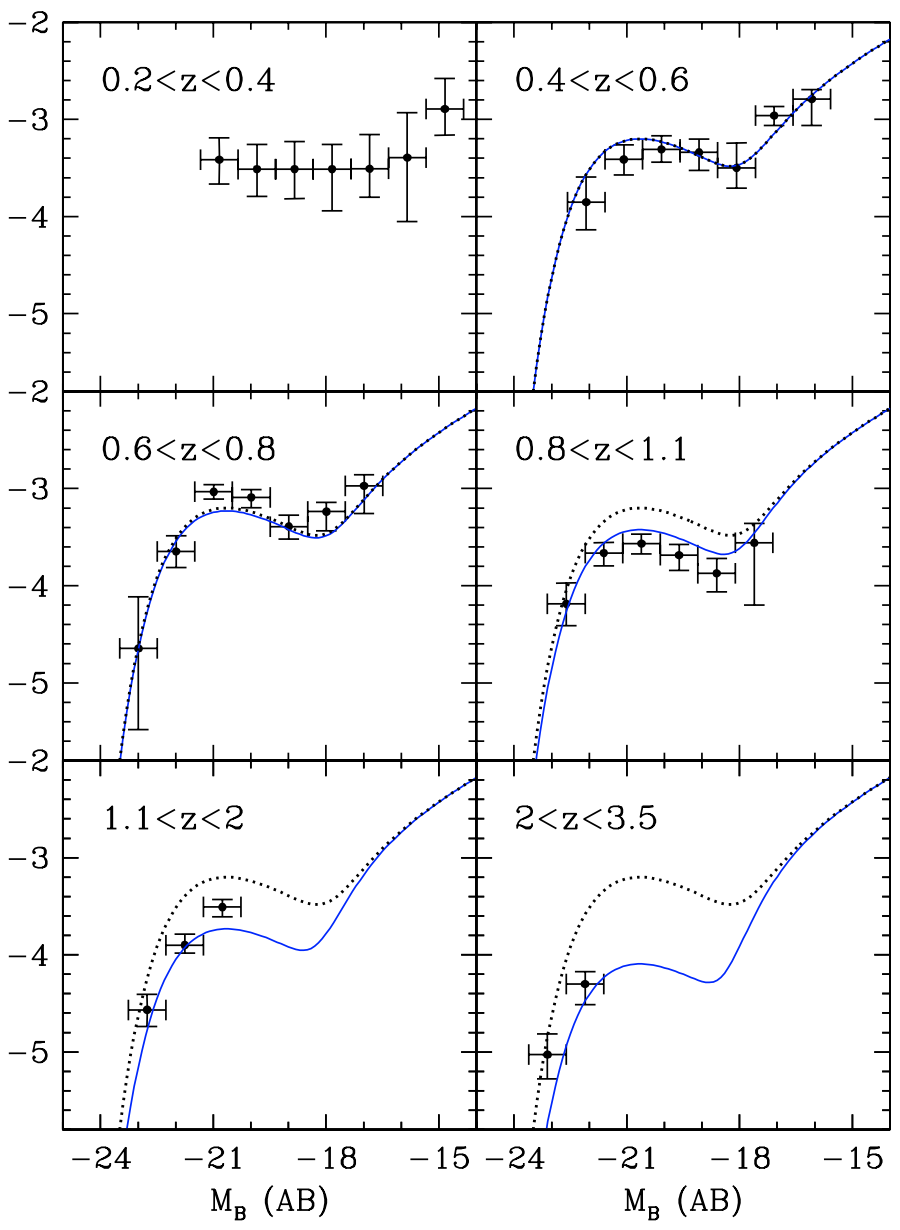

Fig. 9. LFs of the early type galaxies as a function of redshift. The continuous curves comes from our maximum likelihood analysis. The dotted line is our fit at $z \sim 0.5$, reported for comparison in all the redshift bins. The big filled circles are the points obtained with $1 / V_{\text {MAX }}$ method.

with those of a dusty and starburst galaxy. This fraction amounts to $\sim 70 \%$ at $M_{B} \sim-21.5$ and $z \sim 3$.

Finally, the comparison with our hierarchical CDM model shows a slight flattening of the LF at intermediate luminosities. This is because galaxies with larger $\mathrm{M} / \mathrm{L}$ ratios are the main contributors to the red population; these are mainly attained in massive objects (due to the ineffectiveness of gas cooling, to their earlier conversion of gas into stars, and to the effect of AGN feedback) or in low-mass objects (due to the gas depletion originated from the different feedback mechanisms, particularly effective in shallow potential wells). However, the model still overpredicts the LF at faint magnitudes; the origin of such an effect (probably originated at high-redshifts) will be investigated in a future paper.

\subsection{Luminosity densities}

To compare in a global way the redshift evolution of the blue and red galaxies we computed the $B$ band luminosity densities of the two populations as a function of redshift. To make the comparison homogeneous we computed the contribution of the same bright population with $M_{B}(A B) \leq-20.2$ at all redshifts. The results are shown in Fig. 10 for the blue/red and late/early populations. The redshift bins are selected to have a comparable number of objects in the considered magnitude range. In fact for the highest redshift bin the lowest luminosity of the data is

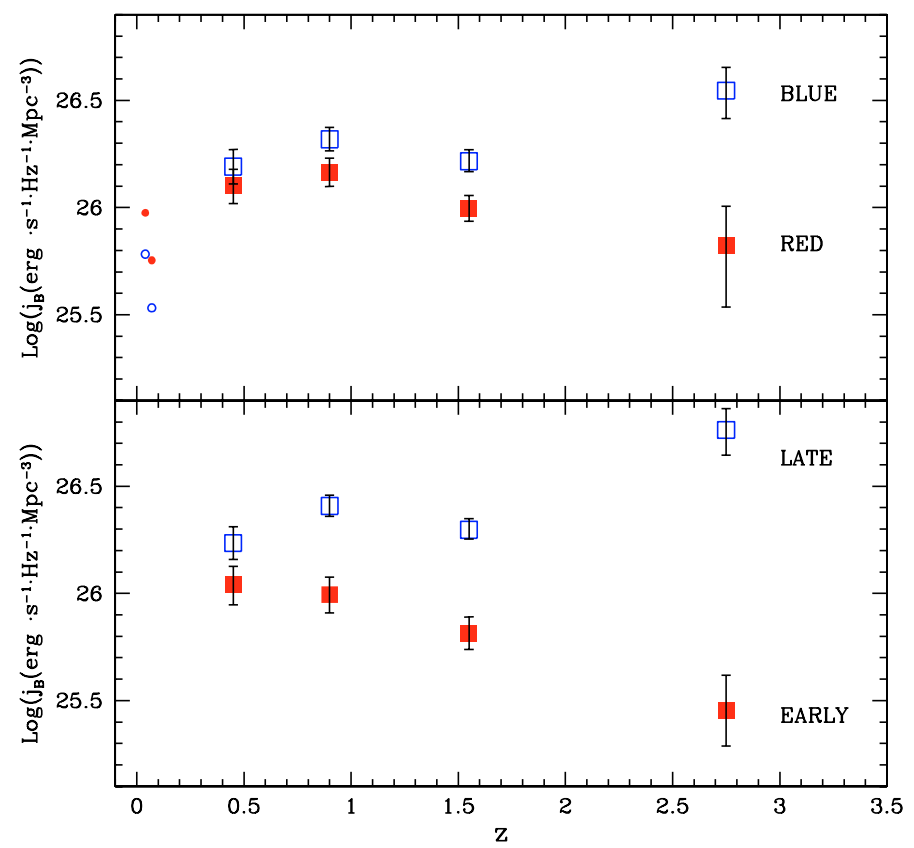

Fig. 10. Upper panel: galaxy luminosity density for red (filled squares) and blue galaxies (empty squares). Lower panel: the same as the upper panel but for early type galaxies (filled squares) and late type galaxies (empty squares). Small points are the local luminosity density estimates by Madgwick et al. (2002) $(z=0.04)$ and Bell et al. (2003) $(z=0.07)$.

$M_{B}(A B)=-21$. For this reason we added the contribution of the fainter sources using the extrapolation of the parametric LF.

The uncertainty associated with the luminosity density is the sum in quadrature of the error estimated through jack$\mathrm{knife}^{1}$ (Efron 1982) analysis, and of the error obtained from the MonteCarlo analysis as described in Sect. 5.1. The first contribution is associated with the clustering properties of the field and the second with the photometric uncertainties. Local luminosity densities, obtained from the integration of the LFs by Madgwick et al. (2002) $(z=0.04)$ and Bell et al. (2003) $(z=0.07)$, are also included for comparison.

The blue/late galaxies increase their luminosity density steadily up to $z \sim 3.5$, while the luminosity density of the red/early population is nearly constant up to $z \sim 1$ and then decreases by a factor $\sim 3$ at $z \sim 3.5$. This confirms our previous result presented in G05, which shows an appreciable decline of the bright red/early population only at $z>1$. As already noted this is not in qualitative contrast with the hierarchical scenario, since the bright and hence massive early population is developing early in the cosmic time in specific overdense regions where the evolution is also accelerated by merging processes. However, the detailed quantitative agreement of the LFs predicted by specific models depends on the details of the main physical processes and a satisfactory agreement is not yet obtained, especially at fainter magnitudes (see previous section).

\section{Red/early faint galaxies}

As shown in the previous section, the presence of an upturn in the LF of the red galaxy population at faint magnitudes is a new feature emerging from the analysis of deep NIR selected galaxies, with respect to previous works at this redshift. To derive

1 The jackknife analysis is performed recomputing the statistic estimate leaving out one observation at a time from the sample. 
hints on the nature of the population responsible for this excess we analysed their colour and spatial properties.

The peculiar shape of the LF, represented by a double Schechter form, appears similar to that obtained for galaxies in local rich clusters. Indeed, recent studies of the total galaxy LFs in clusters selected from the RASS-SDSS survey show an upturn at faint $M_{r}$ that depends on the distance from the cluster centre (Popesso et al. 2006). The main contribution to this local excess comes from the red population selected with $u-r>2.22$ (Strateva et al. 2001). They also noted that the ratio between red and blue galaxies increases with the density in the clusters.

It is interesting to explore whether a dependence on the environment is present for our faint early subsample. To this end, we adopted a 2D density analysis of the 20 first-neighbour method. We describe here briefly the procedure adopted to derive densities, while we refer to Trevese et al. (2007) for a detailed description of the method. A 2D density was assigned to each object and a density map was derived in the field in the redshift interval $z=0.4-0.6$.

A surface density

$\Sigma_{n}=n /\left(\pi D_{p, n}^{2}\right)$

of galaxies was computed considering the projected distances $D_{p, n}$ to the $n$th nearest neighbour, as done by Dressler (Dressler 1980; Dressler et al. 1997). We divide the survey area in cells whose extension depends on the observational accuracy. For each cell we count neighbouring objects at increasing radial distance until a number $n=20$ of objects is reached. In counting galaxies we must take into account the increase of limiting luminosity with increasing redshift for a given flux limit. If $m_{\mathrm{lim}}$ is the limiting (apparent) magnitude in a fixed observing band, at each redshift $z$ we only detect objects brighter than an absolute magnitude $M_{\text {lim }}(z)$, decreasing (brightening) with $z$. We assume the lower limit of the interval as reference redshift $z_{c}$, below which we detect all objects brighter than the relevant $M_{c} \equiv M_{\lim }\left(z_{c}\right)$, which is the magnitude of the faintest galaxy in the $B$ band among the objects we are considering. At $z>z_{c}$ the fraction of detected objects is:

$s(z)=\frac{\int_{-\infty}^{M_{\lim (z)}} \Phi(M) \mathrm{d} M}{\int_{-\infty}^{M_{c}} \Phi(M) \mathrm{d} M}$

where $\Phi(M)$ is the type and z-dependent galaxy $B$-band LF presented above. Thus, in evaluating the galaxies number density, we apply a limiting magnitude correction by assigning a weight $w(z)=1 / s(z)$ to each detected galaxy of redshift $z$. In this way, we have evaluated the surface density field and assigned a density value to each object.

For our analysis we selected galaxies in three magnitude regions: an intermediate region, $-19<M_{B}(A B)<-17$, where the LF of the early population is flat, and two external steeper regions at the bright and faint end of the LF, $M_{B}(A B)<-19$ and $M_{B}(A B)>-17$, respectively.

First of all we note that early galaxies represent the most massive galaxies in each luminosity interval (Fig. 11, panel a). In particular, even at the faint end of the LF the early population is clearly segregated in stellar mass with values one order of magnitude greater on average with respect to the late population.

Looking to the brightest fraction, a clear difference as a function of the density of the environment is found between the early and the late populations. In particular, early galaxies tend to populate regions of higher density. This is shown in Fig. 11, panels $\mathrm{b}$ and $\mathrm{c}$, where the two distributions are represented as
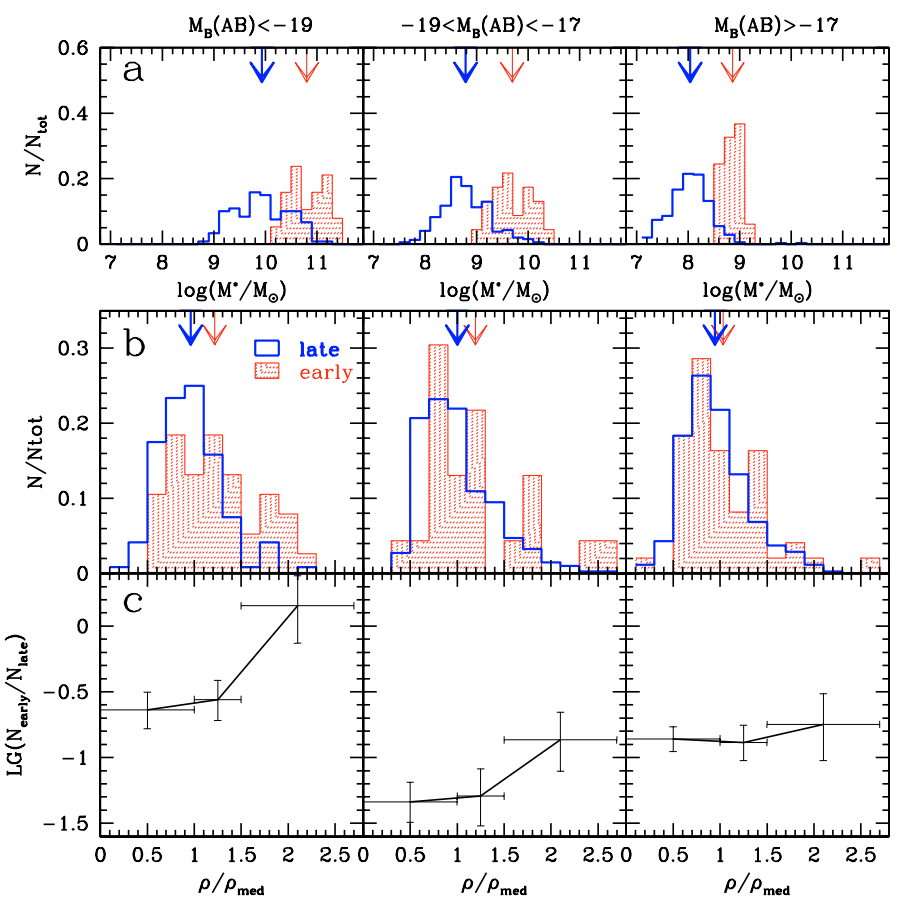

Fig. 11. Panel a): stellar mass distribution for the early and late population, the area of each histogram is normalised to unity. The arrows indicate the mean value of the stellar mass for the early population (thin arrow) and for the late population (thick arrow). Panel b): as for panel a) but for the distribution of $\rho / \rho_{\text {med }}$. Panel c): early type galaxies vs. late type galaxies fraction as a function of the density contrast $\left(\rho / \rho_{\mathrm{ed}}\right)$.

a function of the density of the environment. The ratio early/late increases with density since the average density of the early galaxies is somewhat greater (1.4) with respect to the one of the late population.

This different behaviour becomes less evident with decreasing luminosity and almost disappears at the faint end of the two LFs. We note in this respect that the limited area covered by our sample does not allow an evaluation of the environment dependence up to the high densities that are typical of clusters, like those probed by the Sloan survey for example.

Thus, the scenario that emerges is one where major evolutionary differences between the early and late populations act in the relatively bright galaxies, with $M<-17$ producing the largest differences in the shapes of the two LFs in the interval $-21<M_{B}(A B)<-18$ (flat shape for the early, steep for the late). At faint magnitudes the two LFs tend to converge to the same volume density. From this analysis the characteristic shape of the red/early LF does not seem to depend strongly on the environmental properties.

\section{Summary}

We have used a composite sample of galaxies selected in deep NIR images, obtained from the GOODS public survey, to study the evolution of the galaxy LFs of red/early and blue/late galaxy populations selected using the colour and SSFR statistical properties of the sample.

The observed $U-V$ colour and SSFR distributions show a clear bimodality up to $z \sim 3$, confirming the results obtained in G05 at a higher level of statistical confidence. We found a trend with redshift for the colour magnitude distribution with an intrinsic blueing of about 0.15 mag. in the redshift interval $z=0.4-2.0$ for both populations. This observed bimodality can 
be explained in a hierarchical clustering scenario as being due to the different star formation histories of the red/early and blue/late galaxies (see e.g. Menci et al. 2006).

For the total and the blue/late sample, the LF is well described by a Schechter function and shows a mild luminosity evolution in the redshift interval $z=0.2-1$ (e.g. $\Delta M^{*} \sim 0.7$ for the total sample; $\Delta M^{*} \sim 1$ for the blue/late fraction), while at higher redshifts the LFs are consistent with no evolution. A comparison with our hierarchical CDM model shows a good agreement at bright and intermediate magnitudes. A better agreement of the model was also found at fainter magnitudes due to the suppression of star formation in small objects by the action of an ionising UV background.

The shape of the red/early luminosity function is better constrained only at low and intermediate redshifts and it shows an excess of faint red dwarfs with respect to the extrapolation of a flat Schechter function. In fact a minimum around magnitude $M_{B}(A B)=-18$ is present together with an upturn at fainter magnitudes. This peculiar shape is represented by the sum of two Schechter functions.

We found that the bright one is constant up to $z \sim 0.7$, beyond which it decreases in density by a factor of $\sim 5$ (10 for the early galaxies) up to redshift $z \sim 3.5$. The comparison with our hierarchical CDM model shows that, although the predicted LF has a slight flattening at intermediate luminosity, the model still overpredicts the LF at faint magnitudes. The bright end of the blue and red LFs at low and intermediate redshifts are in good agreement with recent estimates from the DEEP2 spectroscopic survey. As a consequence of this complex evolutionary behaviour, the luminosity densities of the relatively bright $\left(M_{B}(A B)<-20.2\right) \mathrm{red} /$ early and blue/late galaxies show a bifurcation beyond redshift $z \sim 1$. Indeed, the luminosity density of the blue/late population keeps increasing up to $z \sim 3.5$, while the luminosity density of red/early galaxies decreases by a factor of $\sim 2-3$ in the $z=1-3.5$ interval, respectively.

To obtain hints on the nature of the galaxies responsible for the peculiar shape of the red/early LF, we performed an analysis of their stellar masses and spatial distribution. We found that the early galaxies have systematically higher stellar masses, with respect to the late ones, for a given $B$ band luminosity. Brighter early galaxies have a spatial distribution more concentrated in higher density regions when compared to the late ones of the same luminosity class. On the contrary, fainter early and late galaxies show a very similar spatial distribution. Thus, the different environmental properties do not seem to be responsible for the difference in shape at intermediate magnitudes between the blue and red LFs. The latter seems to stem from the different star formation and feedback histories corresponding to different possible merging trees (evolutionary paths) leading to the final assembled galaxy; this specific history, driving the evolution of the star formation, leads to the different $M / L$ ratios characterising the different properties of blue/late and red/early galaxies. In summary, the peculiar shape of the red LF is mainly driven by the nature of the galaxy merging tree rather than by the nurture where the galaxy has grown.

Acknowledgements. We thank the anonymous referee for his/her helpful comments, that led to a significant improvement of the paper. We thank the whole GOODS Team for providing all the imaging material available worldwide. Observations have been carried out using the Very Large Telescope at the ESO
Paranal Observatory under Program IDs LP168.A-0485 and ID 170.A-0788 and the ESO Science Archive under Program IDs 64.O-0643, 66.A-0572, 68.A-0544, 164.O-0561, 163.N-0210 and 60.A-9120.

\section{References}

Arnouts, S., Walcher, C. J., Le Fevre, O., et al. 2007, ArXiv e-prints, 705 Avni, Y., \& Bahcall, J. N. 1980, ApJ, 235, 694

Baldry, I. K., Glazebrook K., Brinkmann J., et al. 2004, ApJ, 600, 681 Baldry, I. K., Balogh, M. L., Bower, R. G., et al. 2006, MNRAS, 373, 469 Bell, E. F., McIntosh, D. H., Katz, N., \& Weinberg, M. D. 2003, ApJS, 149, 289 Bell, E. F., Wolf, C., Meisenheimer, K., et al. 2004, ApJ, 608, 752 Blanton, M. R., Lupton, R. H., Schlegel, D. J., et al. 2005, ApJ, 631, 208 Brown, M. J. I., Dey, A., Jannuzi, B. T., et al. 2007, ApJ, 654, 858 Bruzual, G., \& Charlot, S. 2003, MNRAS, 344, 1000 Cameron, E., \& Driver, S. P. 2007, MNRAS, 377, 523 Castellano, M., Salimbeni, S., Trevese, D., et al. 2007, ArXiv e-prints, 707 Cirasuolo, M., McLure, R. J., Dunlop, J. S., et al. 2007, MNRAS, 380, 585 Cooper, M. C., Newman, J. A., Coil, A. L., et al. 2007, MNRAS, 376, 1445 Cucciati, O., Iovino, A., Marinoni, C., et al. 2006, A\&A, 458, 39

Davis, M., Faber, S. M., Newman, J., et al. 2003, in Discoveries and Research Prospects from 6- to 10-Meter-Class Telescopes II, ed. G. Puragra. Proc. SPIE, 4834, 161

Dekel, A., \& Birnboim, Y. 2006, MNRAS, 368, 2

Dressler, A. 1980, ApJ, 236, 351

Dressler, A., Oemler, A. J., Couch, W. J., et al. 1997, ApJ, 490, 577

Driver, S. P., Allen, P. D., Graham, A. W., et al. 2006, MNRAS, 368, 414

Efron, B. 1982, The Jackknife, the Bootstrap and other resampling plans, CBMS-

NSF Regional Conference Series in Applied Mathematics, Philadelphia:

Society for Industrial and Applied Mathematics (SIAM)

Ellis, R. S. 1997, ARA\&A, 35, 389

Faber, S. M., Willmer, C. N. A., Wolf, C., et al. 2007, ApJ, 665, 265

Fioc, M., \& Rocca-Volmerange, B. 1997, A\&A, 326, 950

Fontana, A., D'Odorico, S., Poli, F., et al. 2000, AJ, 120, 2206

Fontana, A., Pozzetti, L., Donnarumma, I., et al. 2004, A\&A, 424, 23

Fontana, A., Salimbeni, S., Grazian, A., et al. 2006, A\&A, 459, 745

Franceschini, A., Rodighiero, G., Cassata, P., et al. 2006, A\&A, 453, 397

Gabasch, A., Bender, R., Seitz, S., et al. 2004, A\&A, 421, 41

Gabasch, A., Hopp, U., Feulner, G., et al. 2006, A\&A, 448, 101

Gehrels, N. 1986, ApJ, 303, 336

Giallongo, E., D’Odorico, S., Fontana, A., et al. 1998, AJ, 115, 2169

Giallongo, E., Salimbeni, S., Menci, N., et al. 2005, ApJ, 622, 116

Grazian, A., Fontana, A., de Santis, C., et al. 2006a, A\&A, 449, 951

Grazian, A., Fontana, A., Moscardini, L., et al. 2006b, A\&A, 453, 507

Grazian, A., Salimbeni, S., Pentericci, L., et al. 2007, A\&A, 465, 393

Heyl, J., Colless, M., Ellis, R. S., \& Broadhurst, T. 1997, MNRAS, 285, 613

Hogg, D. W., Blanton, M. R., Brinchmann, J., et al. 2004, ApJ, 601, L29

Ilbert, O., Tresse, L., Zucca, E., et al. 2005, A\&A, 439, 863

Ilbert, O., Lauger, S., Tresse, L., et al. 2006, A\&A, 453, 809

James, F., \& Roos, M. 1995, MINUIT Function Mininmization and Error Analysis, version 95.03 (CERN Program Libr. Long Writeup D506; Geneva: CERN)

Labbé, I., Franx, M., Rudnick, G., et al. 2007, ApJ, 665, 944

Madgwick, D. S., Lahav, O., Baldry, I. K., et al. 2002, MNRAS, 333, 133

Marchesini, D., van Dokkum, P., Quadri, R., et al. 2007, ApJ, 656, 42

Mateus A., Sodré, L., Cid Fernandes, R., \& Stasińska, G. 2007, MNRAS, 374, 1457

Menci, N., Fontana, A., Giallongo, E., \& Salimbeni, S. 2005, ApJ, 632, 49

Menci, N., Fontana, A., Giallongo, E., Grazian, A., \& Salimbeni, S. 2006, ApJ, 647, 753

Norberg, P., Cole, S., Baugh, C. M., et al. 2002, MNRAS, 336, 907

Pentericci, L., Grazian, A., Fontana, A., et al. 2007, A\&A, 471, 433

Poli, F., Giallongo, E., Fontana, A., et al. 2003, ApJ, 593, L1

Popesso, P., Biviano, A., Böhringer, H., \& Romaniello, M. 2006, A\&A, 445, 29

Pozzetti, L., Cimatti, A., Zamorani, G., et al. 2003, A\&A, 402, 837

Sandage, A., Tammann, G. A., \& Yahil, A. 1979, ApJ, 232, 352

Somerville, R. S., \& Primack, J. R. 1999, MNRAS, 310, 1087

Strateva, I., Ivezić, Ž., Knapp, G. R., et al. 2001, AJ, 122, 1861

Trevese, D., Castellano, M., Fontana, A., \& Giallongo, E. 2007, A\&A, 463, 853

Trujillo, I., Förster Schreiber, N. M., Rudnick, G., et al. 2006, ApJ, 650, 18

Weiner, B. J., Phillips, A. C., Faber, S. M., et al. 2005, ApJ, 620, 595

Willmer, C. N. A., Faber, S. M., Koo, D. C., et al. 2006, ApJ, 647, 853

Wolf, C., Meisenheimer, K., Rix, H. W., et al. 2003, A\&A, 401, 73 